\title{
Archives of Clinical and Biomedical Research
}

Volume 1, Issue 2

Research Article

\section{Laboratory Patterns in Hypoglycin A Toxicity}

\author{
Fabian Pitkin*, Sala Randall \\ Department of Medical Technology, College of Natural and Applied Science, Allied Health and Nursing, Northern \\ Caribbean University, Manchester Road, Mandeville, Jamaica \\ *Corresponding Author: Fabian Pitkin, Department of Medical Technology, College of Natural and Applied \\ Science, Allied Health and Nursing, Northern Caribbean University, Manchester Road, Mandeville, Jamaica, E-mail: \\ fabian.pitkin@ncu.edu.jm
}

Received: 21 March 2017; Accepted: 03 April 2017; Published: 10 April 2017

\begin{abstract}
The unripe fruit of the ackee tree (Blighia Sapida), contains significantly high concentrations of the hypoglycin (L- $\alpha$ amino- $\beta$-[methylene cyclopropyl] propionic acid) a toxin implicated in over 5000 deaths worldwide. Methylenecyclopropane acetyl CoA (MCPA-CoA) a metabolite of hypoglycin A, inhibits several coenzyme A dehydrogenases which are essential for gluconeogenesis resulting in depletion of glucose reserves and the inability of cells to regenerate glucose; eventually leading to hypoglycaemia. This study aimed at reviewing literature on the hypoglycin A toxicity, add to the body of tests already established by the WHO in detecting hypoglycin toxicity based on the patterns we have observed and to provide physicians with a simple panel of tests that will aid in the speedy detection of hypoglycin A toxicity for more timing therapeutic intervention. This review covers the description, pathophysiology, epidemiology, and clinical presentation.

In addition, data on patients diagnosed with hypoglycin A toxicity was collected and analysed for significant trends. Analysis of data determined that $86 \%, 97 \%$ and $79 \%$ of patients demonstrated significant increase in phosphorous (95\% CI of 1.74-2.73), anion gap (95\% CI 24.26-29.05) and AST (95\% CI 37.97-73.06) respectively. On the other hand, $95 \%$, and $92 \%$ of patients demonstrated significant decrease in bicarbonate (95\% CI of 13.02-16.56) and Lymphocytes count 95\% CI of 7.49-12.81 respectively. Significantly low random plasma glucose levels, in conjunction with elevated levels of Phosphorus, Anion gap, and AST, as well as decreased levels of bicarbonate, and an absolute lymphocyte count could prove an effective screening for Hypoglcyin A toxicity in affected regions.
\end{abstract}


Keywords: Ackee; Hypoglycin A; Jamaican vomiting sickness; Laboratory profile

\section{Introduction and Review of Literature}

The ackee fruit (Blighia sapida) has its origins in West Africa and is indigenous to the Caribbean. Consumption of the fruit is mainly in Haiti, some parts of West Africa, and Jamaica- where ackee is a component of the national dish. The fruit is composed of a pod, the seed, and the aril (the part which is consumed). Ackee should be allowed to open and ripen naturally on the tree, exposing the black seeds and the yellow arils. In 1954, Hassal et al were the first to isolate 2 toxic compounds in their crystalline form. These compounds were called hypoglycin A and hypoglycin B because of their hypoglycaemic activity [1]. Of the two toxic compounds however, only hypoglycin A can be found in the aril of the fruit. "Hypoglycin A is found predominantly in the immature fruit. Concentrations of hypoglycin A within the arilli ranges from over $1000 \mathrm{ppm}$ in the immature fruit to less than $0.1 \mathrm{ppm}$ in the fully mature fruit. Ill effects occur only when the immature fruit is consumed" according to Goldson [2].

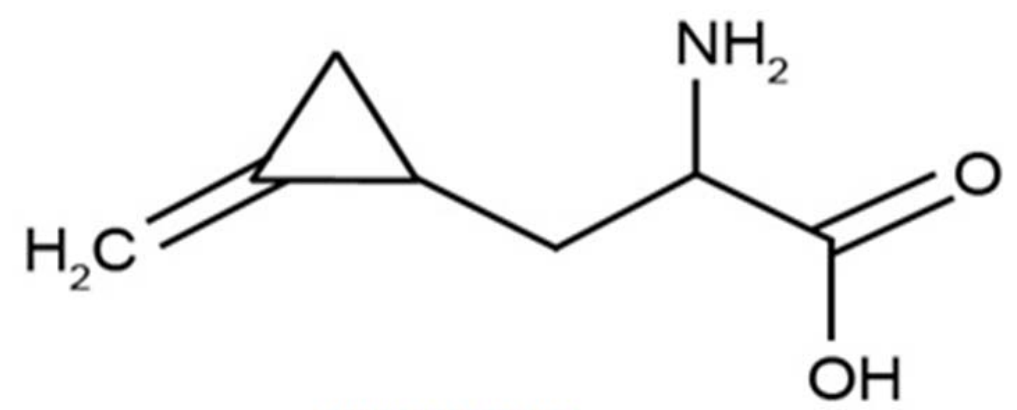

HYPOGLYCIN A

Hypoglycin A (L- $\alpha$-amino- $\beta$-[methylene cyclopropyl] propionic acid) is a toxic amino acid who's metabolite (methylenecyclopropane acetyl CoA/ MCPA-CoA) according to Holson et al. [1] is the causative agent of Jamaican Vomiting Sickness (JVS). MCPA-CoA exerts its effect by inhibiting several coenzyme A dehydrogenases which are essential for gluconeogenesis resulting in depletion of glucose reserves and the inability of cells to regenerate glucose; eventually leading to hypoglycaemia. Goldson [2] states that altered levels of circulating insulin do not cause hypoglycaemia associated with hypoglycin action. An association between ackee poisoning and Jamaican vomiting sickness was first noted in 1875 and documented in 1904 [1].

Symptoms of ackee poisoning or Jamaica vomiting sickness include uncontrollable vomiting, diarrhoea, abdominal cramps, and drowsiness. In some cases, the patient becomes comatose and death may follow [3]. Once the sickness Arch Clin Biomed Res 2017; 1 (2): 76-84 
begins, symptom progression is rapid. Patients experience pronounced diaphoresis, tachypnea, tachycardia, headache, generalized weakness, paraesthesia, and disturbed mental states. After a period of prostration, which may last as long as 18 hours, a second bout of vomiting may occur. Unless treatment is given, this episode is usually followed by convulsions, coma, and death [1].

The laboratory plays a major role in the confirmation of hypoglycin toxicity. According to Holson [1], patients suspected of having ackee fruit poisoning should immediately do a finger stick glucose test, due to its urgency it is not recommended to wait for serum glucose results. In addition, Holson suggest expected abnormalities in serum electrolytes, liver transaminases, and urinalysis in persons experiencing hypoglycin toxicity. Goldson [2] also notes urine abnormality linking deaths to ackee intoxication due to enhanced concentrations of dicarboxylic acids in the urine of the victims. Barceloux et al. [4] also suggests the need to test serum glucose, serum electrolytes, arterial $\mathrm{pH}$, serum aspartate aminotransferases, and serum creatinine in the detection and monitoring of ackee poisoning due to the change in behaviour of these analytes as a result of this condition.

In addition to severe hypoglycaemia, serious intoxication laboratory abnormalities include metabolic acidosis, electrolyte imbalance (e.g. hypokalemia), and laboratory evidence of dehydration (e.g. elevated serum BUN to creatinine ratio) [4]. Increases in the leukocyte count often occur in acute poisoning, for example, in response to an acute metabolic acidosis [5]. The clinical laboratory also plays a significant role in the preparation of post mortem histological samples where depleted glycogen stores and fatty metamorphosis of organs (eg. liver, kidneys) is noted [1].

\section{Methodology}

A retrospective descriptive design was used to identify patterns in laboratory data in patients $(\mathrm{n}=33)$ diagnosed as having had hypoglycin A toxicity. Data was obtained from a major hospital in western Jamaica following an outbreak of ackee poisoning in the region. Results were recorded for each patient with major emphasis on U\&Es (BUN, creatinine, and electrolytes), liver function tests (LFTs), and complete blood count (CBC). Other test results obtained include uric acid, amylase, CPK, and CK-MB. The electrolyte values were used to determine anion gap (AG) for each patient. 
After data was collected and tabulated, each analyte was assessed for any apparent trends in proportions of elevated, normal, and decreased when compared to the reference ranges. Standard descriptive statistics were used to assess baseline characteristics. Continuous data corresponding with the various analytes were represented using box and whisker plots, as well as histograms developed by use of the SPSS version 20. The significance of the various trends obtained was strengthened by determination of the $95 \%$ confidence interval for the analytes of interest.

\section{Results}

A total of thirty three (33) patient results of those diagnosed as having had ackee poisoning were analysed as five (5) were excluded from the study due to missing or incomplete data. Among the thirty three (33) sets of results analyzed, $86 \%, 97 \%$ and $79 \%$ of patients demonstrated significant increase in phosphorous (95\% CI of $1.74-2.73$ ), anion gap (95\% CI 24.26-29.05) and AST (95\% CI 37.97-73.06) respectively (Figures 1, 2 and 3). On the other hand $95 \%$, and $92 \%$ of patients demonstrated significant decrease in bicarbonate (95\% CI of 13.02-16.56) and Lymphocytes count respective 95\% CI of 7.49-12.81 respectively (Figures 4 and 5).
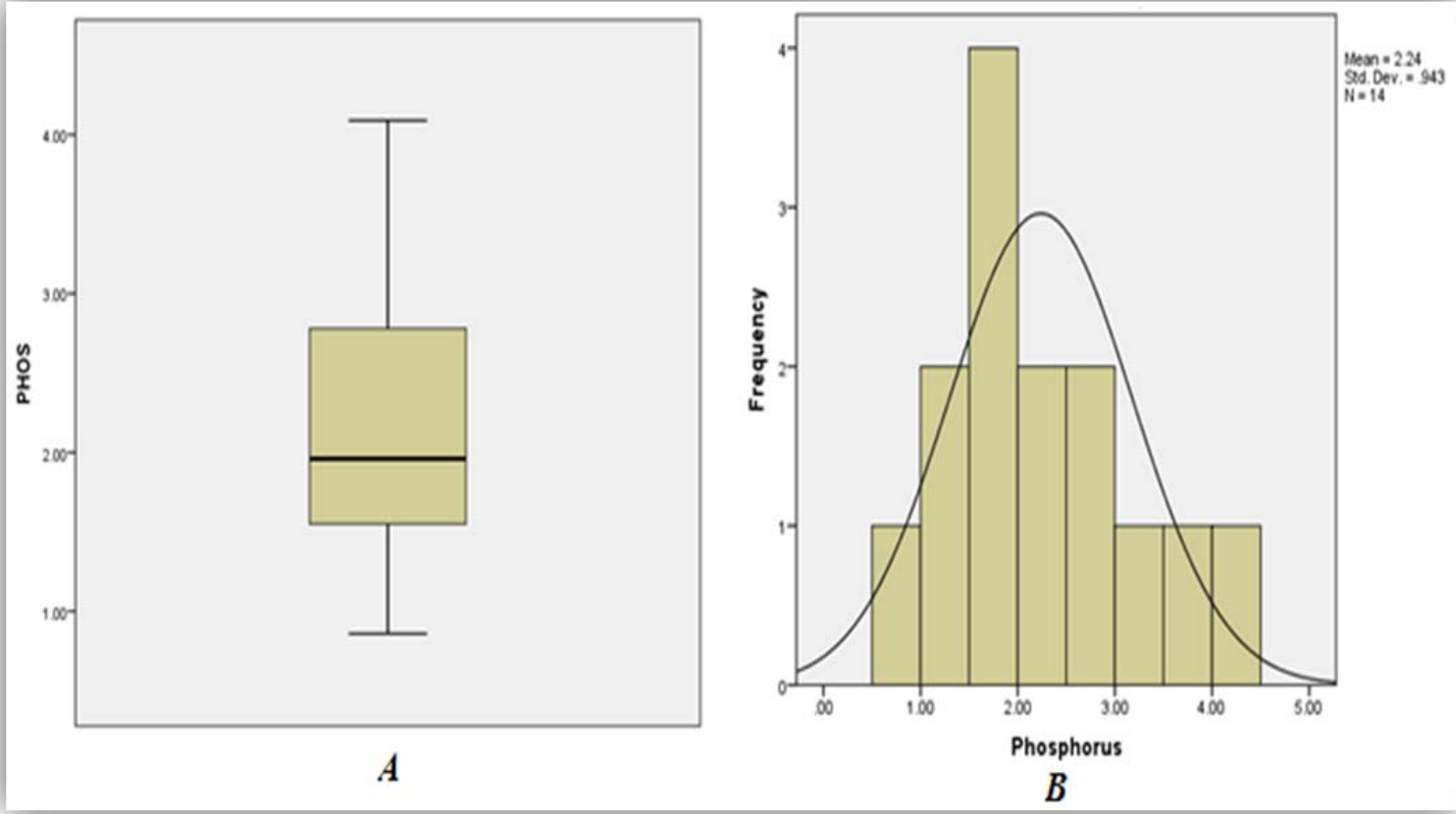

Figure 1: Effects of Hypoglycin A on patients serum phosphorus levels primarily associated with significantly high levels of phosphorus in the unripe ackee. 


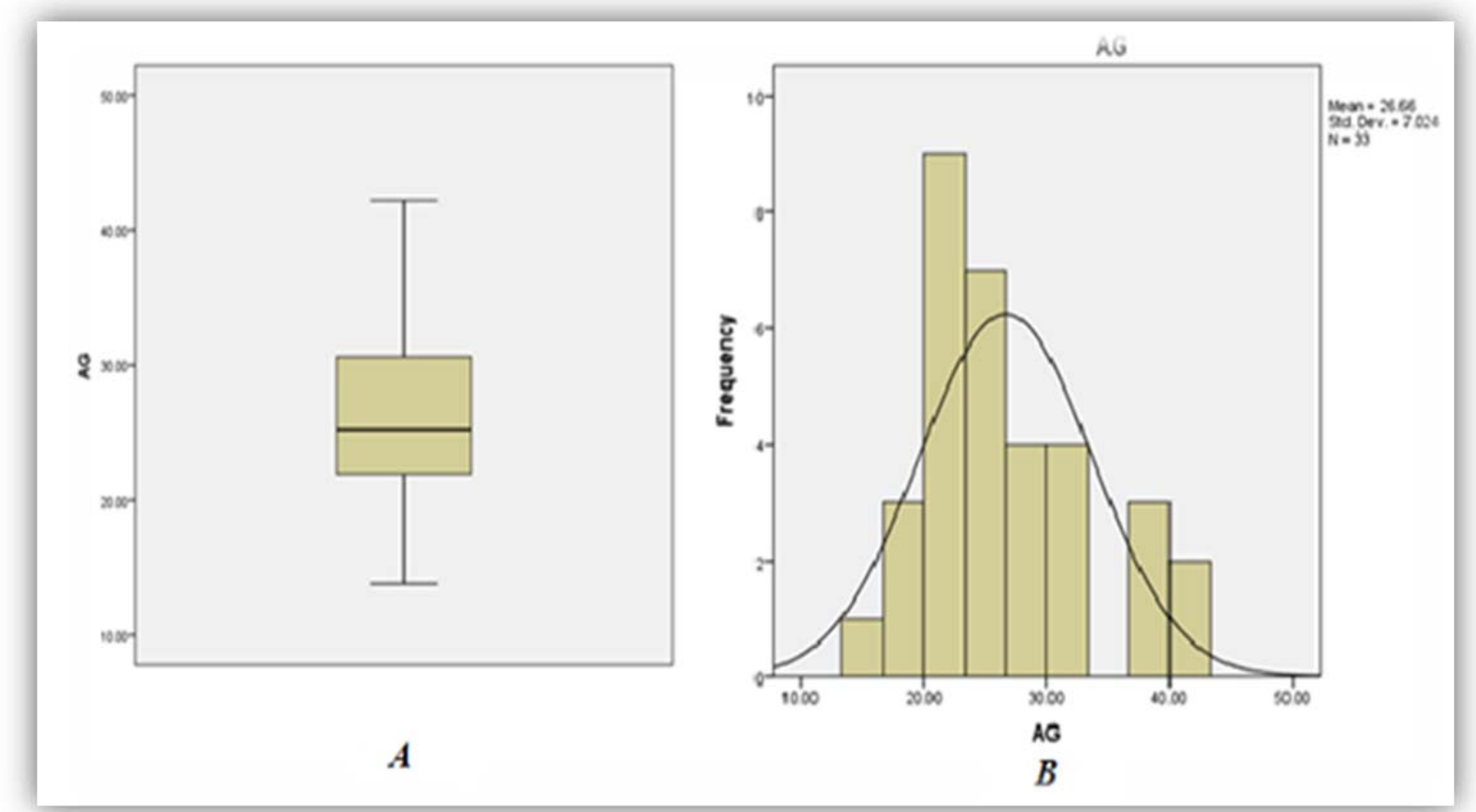

Figure 2: The effects of Hypoglycin A on patients Anion gap directly associated with pathophysiological change in electrolyte balance.

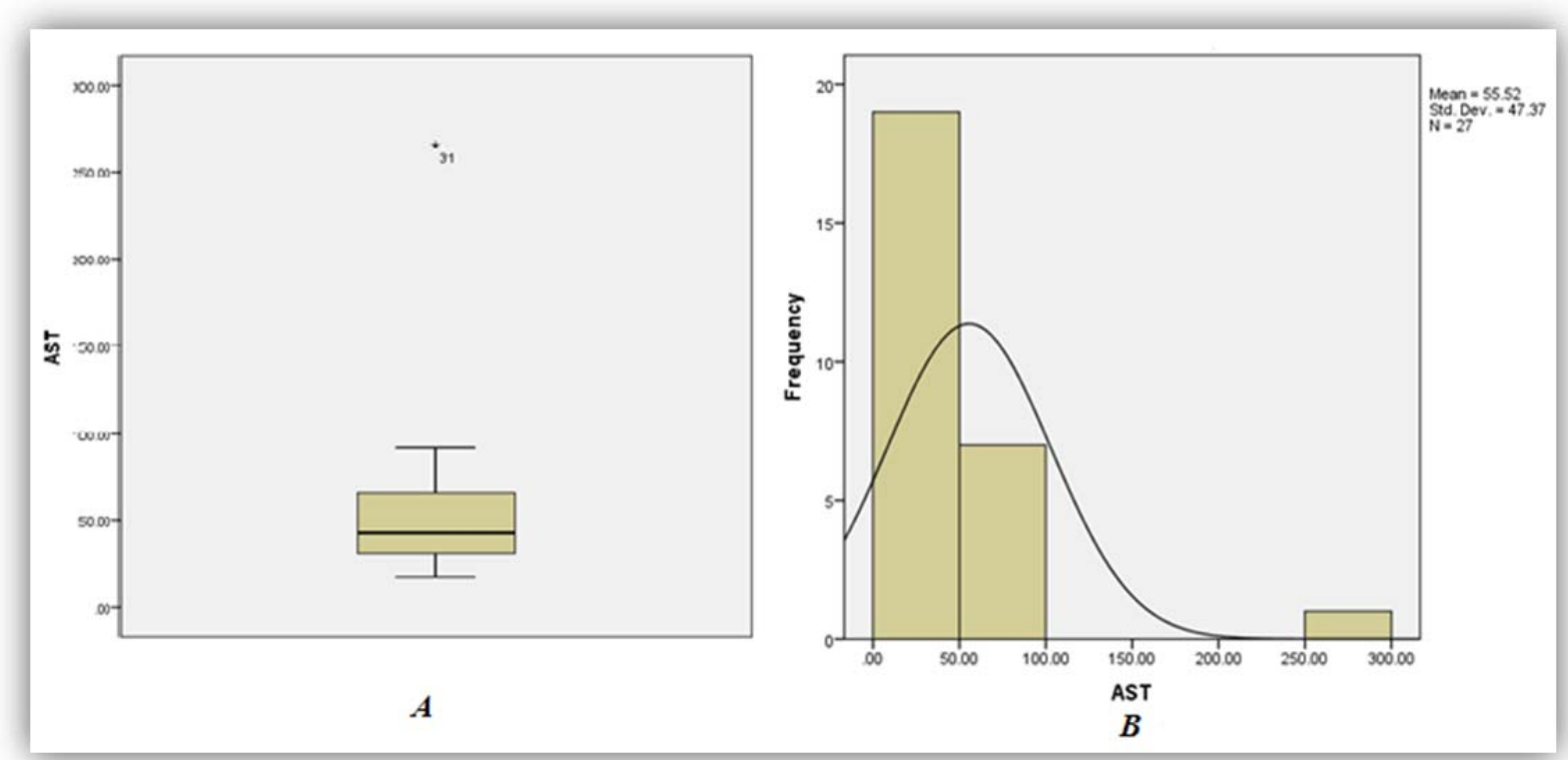

Figure 3: Effects of Hypoglycin A on patients' liver demonstrated by significant changes in serum aspartate aminotransferases. 

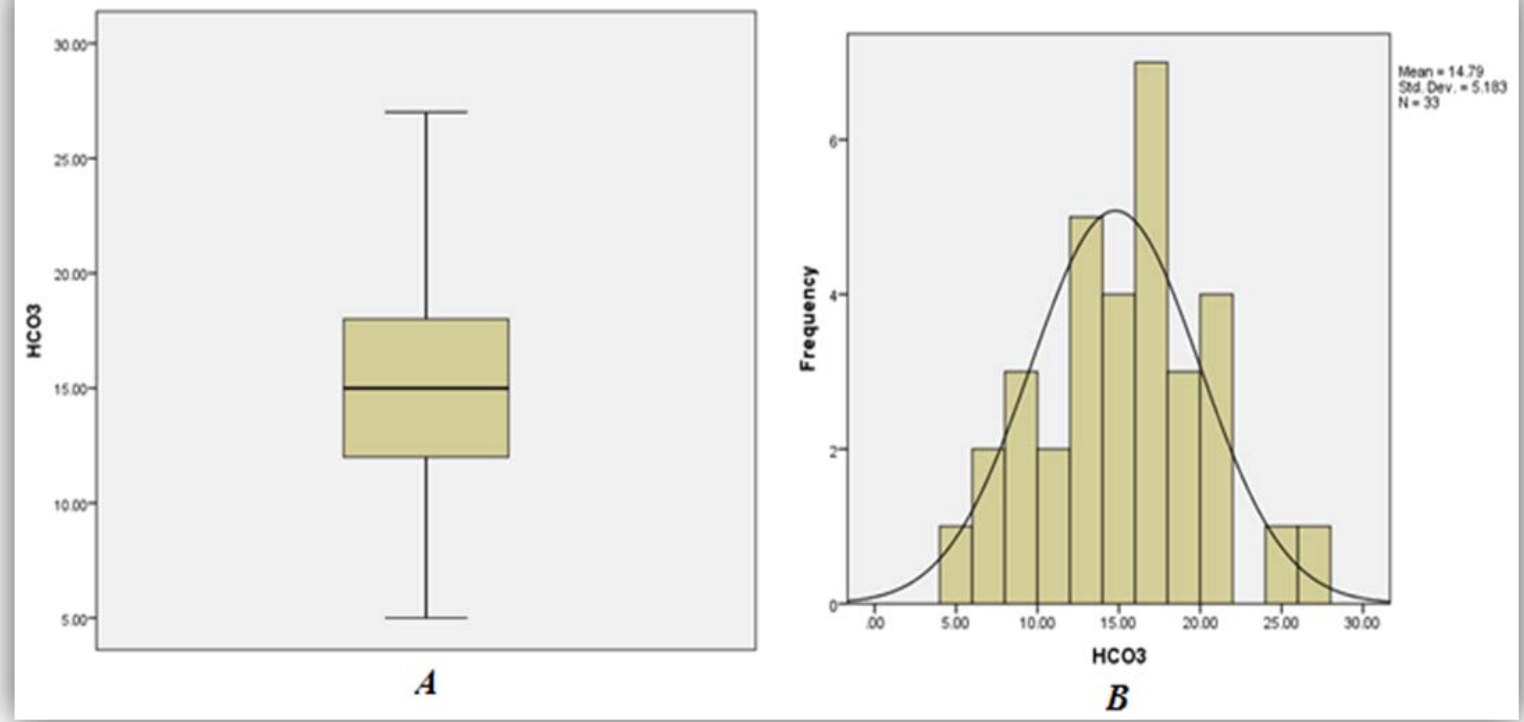

Figure 4: Effects of Hypoglycin A on patients acid-base balance demonstrated by significant changes in serum Bicarbonate measurements.
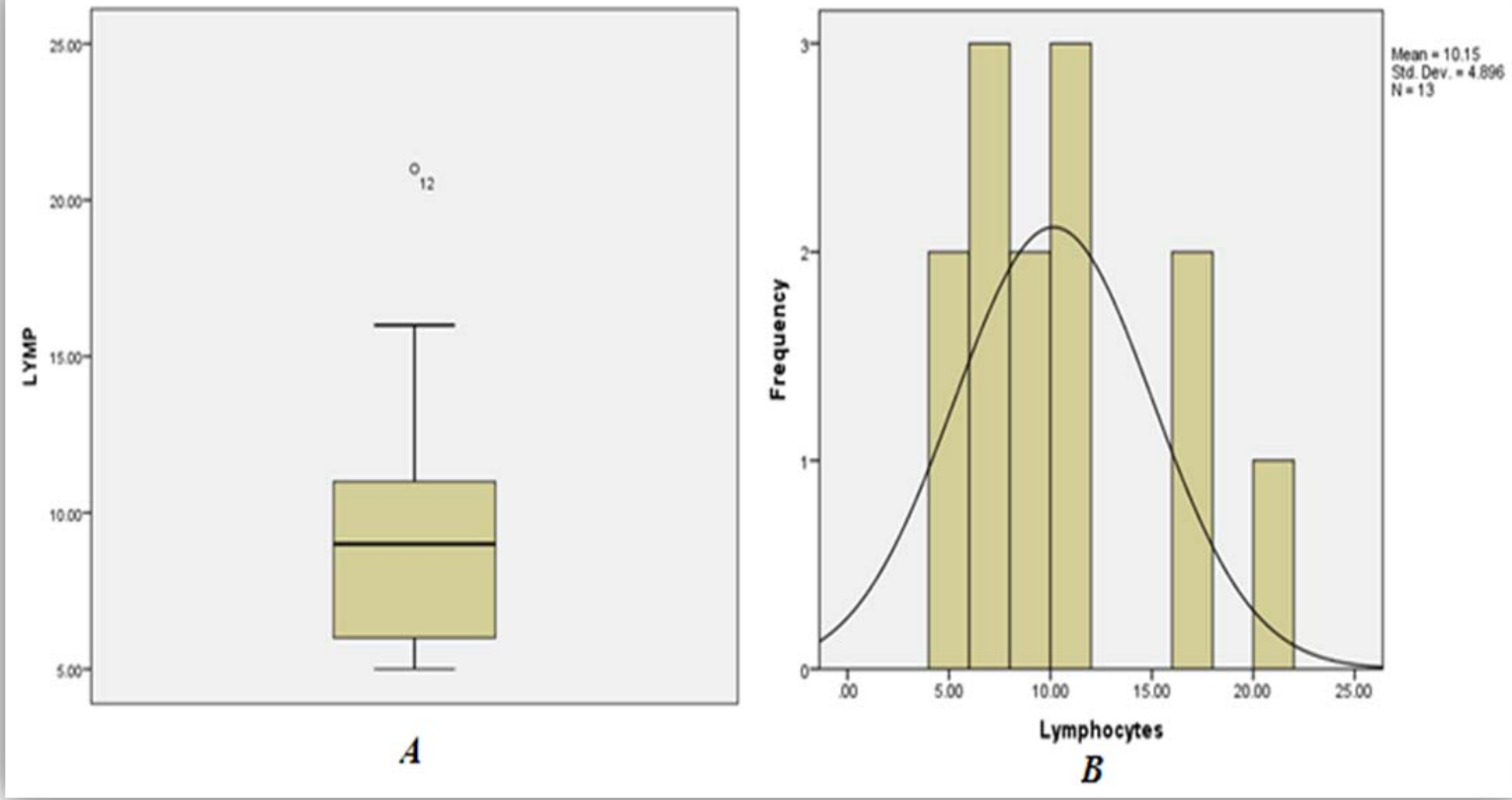

Figure 5: Effects of Hypoglycin A on patients immune response demonstrated by significant changes in white blood cell distribution with a notable reduction in relative lymphocytes count. 


\section{Discussion}

As seen in Table 1 above, $86 \%$ of patients involved in the study had increased levels of serum phosphorus consistent with the high concentration of phosphorus to be found in the unripe fruit. An increased trend was also observed for anion gap and the decreased levels of bicarbonate are consistent with the hyperemesis induced by the toxic process. As with most toxic processes the liver is affected evidence by increased concentrations of AST. The relative lymphocyte count was decrease in $92 \%$ with no clear association with the toxicity. It is the researchers' belief that the toxins may suppress the proliferation of the cell line and or facilitate neutrophilia resulting in altered distribution.

\begin{tabular}{|l|c|c|c|c|c|}
\hline \multicolumn{1}{|c|}{ Analyte } & $\begin{array}{c}\text { Percentage } \\
\text { Normal }\end{array}$ & $\begin{array}{c}\text { Percentage } \\
\text { Increased }\end{array}$ & $\begin{array}{c}\text { Percentage } \\
\text { Decreased }\end{array}$ & $\mathbf{9 5 \% ~ C I ~}$ & Reference range \\
\hline Phosphorous & 7 & 86 & 7 & $1.74-2.73$ & $0.96-1.40(\mathrm{mg} / \mathrm{dL})$ \\
\hline Lymphocyte (Relative Count) & 8 & 0 & 92 & $7.49-12.81$ & $20-55(\%)$ \\
\hline Anion Gap & 3 & 97 & 0 & $24.26-29.05$ & $8-16(\mathrm{mOsm} / \mathrm{Eq})$ \\
\hline Bicarbonate & 9 & 0 & 91 & $13.02-16.56$ & $21-28(\mathrm{mmol} / \mathrm{L})$ \\
\hline AST & 21 & 79 & 0 & $37.97-73.06$ & $10-30(\mathrm{IU} / \mathrm{L})$ \\
\hline
\end{tabular}

Table 1: The pattern of increase or decrease in patient values, coupled with the $95 \%$ confidence intervals (CI) presented against the reference ranges for each.

The wide spread use of ackee in Jamaica naturally increases the risk for hypoglycin A toxicity. Another factor that inadvertently increases the risk of succumbing to the toxic effects of the fruit includes: large scale harvesting of the unripe fruits by individuals who are not altogether careful to store and prepare the fruits in such a manner that renders it less toxic. While much effort has been made to educate the wider society about the toxic effects of harvesting the unripe fruit, the practice has become more prevalent as the demand for the fruit increases.

It is important to identify laboratory pattern(s) in hypoglycin A toxicity as it serves to guides interventions especially in cases where there is no definitive knowledge or evidence of ackee consumption. Understanding the patterns will 
equip clinicians with the tool to assist patients in more severe cases of the toxicity for example in a hypoglycin A induced hypoglycaemic coma.

The chemical detection of Hypoglycin A and it's by-products in Jamaica and the Caribbean on a whole could prove very time consuming as most clinical laboratories are not equipped with the requisite automated instruments to detect this toxic agent.

For the diagnosis of hypoglycin A, timely blood specimen must be collected from suspected patient(s) and delivered to an approved testing facility which does quantitative analysis of the toxin. Several testing methods are employed in the quantitative analysis of hypoglycin A for example High Performance Liquid Chromatography (HPLC) and PicoTag method none of which are available in clinical laboratory used for routine assessment of patient status. It is therefore necessary for clinicians to be equipped with knowledge of the common trends/ patterns consistent with hypoglycin A toxicity derived from routine tests with a quicker turn-around time than the more complex and costly quantitative methods of analysis.

\section{Limitations}

The absence of other patient demographics limited the study to analyses of result only. The sample size analyse were small. A more robust data set would have allowed for a better observation of patterns. While there were thirty eight sets of patient results only thirty five were analyzed due to missing data.

\section{Conclusion}

Ackee is an indigenous plant of Jamaica and is widely used in local dishes, especially as it is a major component of Jamaica's signature dish. The ingestion of the unripe fruit exposes individuals to fatal concentrations of hypoglycin A and as such indiscriminate sale and consumption of unripe fruits should be discontinued. While the direct detection of hypoglycin A presents a challenge, diagnosis can be facilitated from the clinical laboratory perspective based on consistent laboratory patterns seen in actual cases. A review of laboratory data reveals a useful pattern where AST, phosphorus and anion gap are significantly increased with a notable decrease in bicarbonate and relative lymphocyte count. When assessed, these analytes could aid in early detection and lifesaving intervention. Further studies will be required to validate these patterns in other geographical locations. 


\section{References}

1. Holson D, Corden E. Ackee Fruit Toxicity. eMedicine. Retrieved 2/28/2014 from http://emedicine.medscape.com/article/1008792.

2. Goldson A. The ackee fruit (Blighia sapida) and its associated toxic effects. The Science Creative Quarterly 2 (2007).

3. Golden KD. Hypoglycin: a toxic amino acid of the ackee plant. Caribbean Poison Information Network (CARPIN) First Scientific Conference June 3-4, 2006.

4. Barceloux D. (2008). Medical toxicology of natural substances. John Wiley \& Sons, Inc., Hoboken, New Jersey

5. Dart R, et al. Medical Toxicology. 3rd edition Lippincott Williams \& Wilkins. ISBN-13 (2014): 9780781728454 